\title{
Diffuse Interstellar Band Profiles
}

\author{
P. J. Sarre \\ School of Chemistry, The University of Nottingham, \\ University Park, Nottingham, NG7 2RD, United Kingdom \\ email: Peter.Sarre@Nottingham.ac.uk
}

\begin{abstract}
A short review of the profiles of diffuse interstellar bands and their possible interpretation is given, largely from the perspective of absorption by gas-phase molecules. Potentially a crucial source of information on the nature of the diffuse band carriers, profile studies provide a promising avenue of research towards finding the solution to their identification. Detection and modelling of fine structure in particular offers a valuable benchmark against which any proposed carriers(s) can be tested.
\end{abstract}

\section{Introduction}

Interest in the shapes of the diffuse interstellar absorption bands stretches over many decades. Even in the early photographic spectra of Mary Lea Heger, recently digitised by McCall \& Griffin (2013), the difference in width of $\lambda 5780$ and $\lambda 5797$ is clear. On the commencement of systematic studies Merrill (1934) wrote: 'Instead of being narrow and sharp, however, as interstellar lines should be, they are somewhat widened and have rather diffuse edges.' Characteristics such as bands being 'V-shaped' or 'asymmetric' were discussed in some detail in a key review by Herbig (1975). Some examples of the range of widths and shapes seen in spectra he recorded towards HD 183143 are illustrated in Fig. 1. For example, the widths of $\lambda 6195$ and $\lambda 6177$ or $\lambda 5780$ and $\lambda 5797$ may be compared.

The strongest and broadest diffuse band is $\lambda 4428$ (formerly called $\lambda 4430$ ), with a typical equivalent width of a few $\AA$, FWHM of $c .17 \AA$ and depth of $11-18 \%$ towards Cyg OB2 stars with $\mathrm{E}_{B-V}$ values in the range 1-2 (Snow, Zukowski \& Massey 2002). This band alone is therefore responsible for the removal of a large amount of starlight and it received most of the attention until the 1970s when growth in observations of narrower features began. For a summary of studies of $\lambda 4428$ to 1975 see Herbig (1975) and for the 50th anniversary celebrations of its discovery see Bromage (1987). Although very broad bands such as $\lambda 4428$ and $\lambda 6177$ are clearly extremely important, other than through a flash of inspiration or a serendipitous experiment it is not easy to devise a strategy for identification of the carriers of such wide features. However, some of narrower bands do offer a route that can be pursued, particularly since the discovery of fine structure. First, the various possible contributions to the observed spectral widths and shapes are outlined.

\section{Band linewidth and shape}

Whether the carriers are free gas-phase molecules or dust grains, or indeed something that would be considered intermediate between these limiting cases, the Doppler spread arising from the kinetic temperature and turbulence in a single cloud contribute to the line width, with further broadening if there is more than one cloud along the line-ofsight. As the rest frequencies for the 'diffuse band' transitions are unknown, a reference 

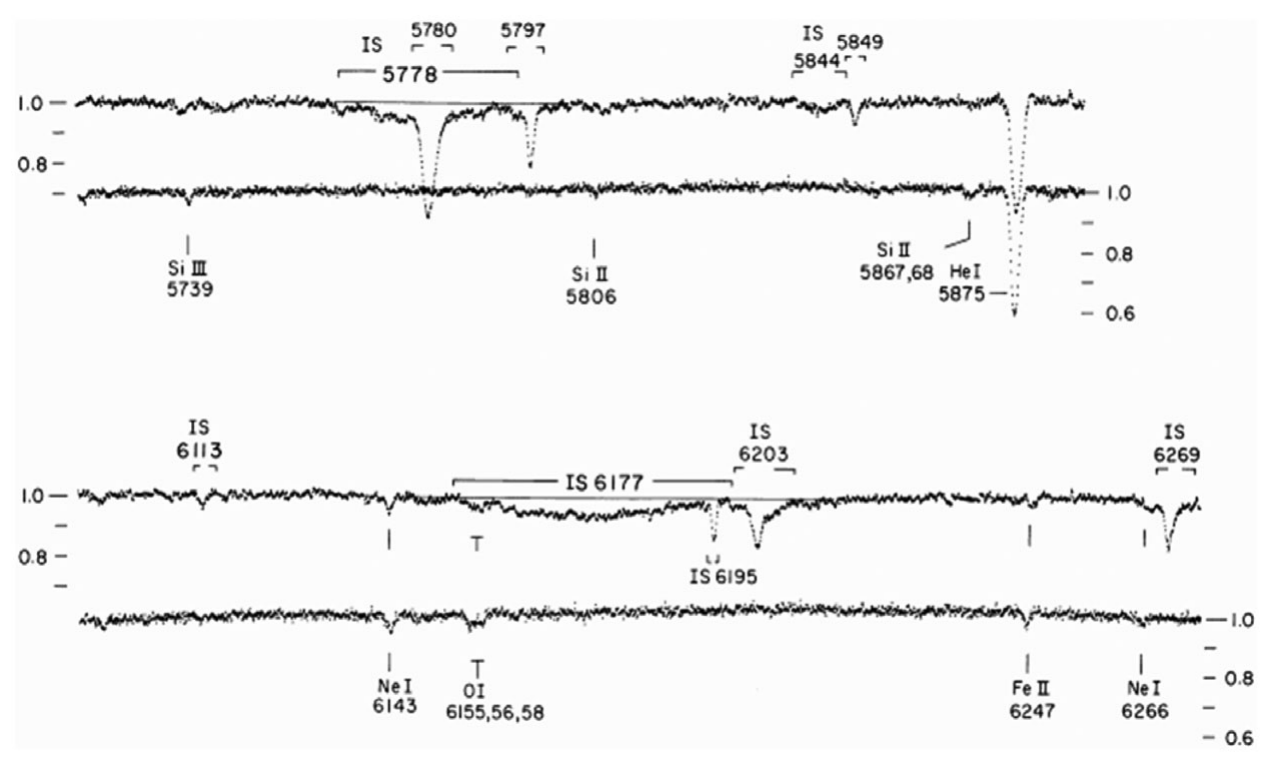

Figure 1. Comparison of spectra recorded towards the reddened star HD 183143 (upper of each pair of traces) with an unreddened standard (lower). 'IS' indicates diffuse bands of interstellar origin. Note the He I photospheric line that appears in the spectra of both stars which are of similar type. Reproduced with permission from Herbig (1975).

is required and is usually afforded by recording electronic absorption lines of e.g. K I or the $\mathrm{CH}$ molecule (when present). For diffuse band profile studies considerable effort is made to select 'single cloud' lines-of-sight in order to minimise undesirable complexity arising from overlapping velocity components.

For gas-phase molecules the band width is normally considered to arise principally from the spread of rotational lines which in the simplest case would be due to $\mathrm{P}(\Delta \mathrm{J}=-1)$ and $\mathrm{R}(\Delta \mathrm{J}=+1)$ rotational branches where $\mathrm{J}$ is the molecular rotational quantum number. For large molecules a set of rotational lines arises even in cold diffuse clouds on account of the high moment of inertia of the carrier molecule and resultant closely spaced rotational levels. For some electronic transitions a $\mathrm{Q}(\Delta \mathrm{J}=0)$ branch can also appear and tends to be sharper than the $\mathrm{P}$ or $\mathrm{R}$ branches. Excitation of an electron generally causes a relaxation in the molecular geometry and a consequent increase in one or more of the moments of inertia. This scenario can result in the formation of a rotational branch head which for most cases leads to a short-wavelength spectral limit in the R-branch - or a 'steep blue side' for the profile; the converse is also known, one such example being the Swan bands of the $\mathrm{C}_{2}$ molecule. Diffuse interstellar bands with a 'steep blue side' are quite common. The spectral complexity expands further when the molecule has an open-shell electronic structure.

Each rotational line has an intrinsic width resulting from the lifetime of the excited state level which could be the natural lifetime towards radiative decay or significantly shorter due to intramolecular interactions which may be J-, v- and energy-dependent. Such internal vibrational redistribution (IVR) processes are common in large molecules. Broadening of excited state levels could also arise from predissociation or autoionisation but this is now largely discounted as the carrier is destroyed on photon absorption, necessitating rapid chemical re-formation processes which are improbable in the interstellar context. 


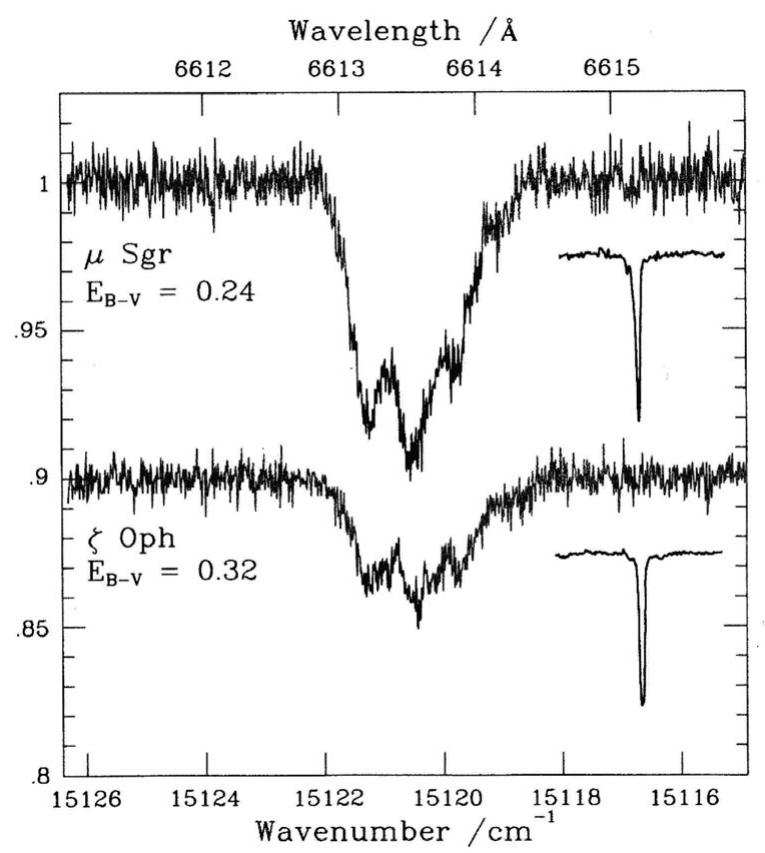

Figure 2. Fine structure detected in a diffuse interstellar band $(\lambda 6614)$ recorded with the UHRF at the Anglo-Australian Observatory towards $\mu \mathrm{Sgr}$ and $\zeta$ Oph at RP $=600,000$. The K I line (recorded at $\mathrm{RP}=300,000$ ) is shown for comparison and is scaled to the same dispersion at the diffuse band wavelength. The wavenumber and wavelength values are both given in air. Reproduced with permission from Sarre et al. (1995).

\section{Observations and modelling of diffuse band profiles}

Observations of $\lambda 5780$ (Savage 1976) and $\lambda 6614$ (Welter \& Savage 1977) at a resolving power of $c .30,000$ have been published, the latter work revealing a steep short-wavelength side for $\lambda 6614$. In a significant advance the first attempt to reproduce a diffuse band profile $(\lambda 5780)$ through rotational contour modelling of electronic transitions in molecules was reported by Danks \& Lambert (1976). A first indication of possible structure in a diffuse band ( $\lambda 6614)$ was found by Herbig \& Soderblom (1982) in the form of a small inflection on the side of the band as seen towards HD 187982 and HD 186745; this feature, viewed in retrospect, is probably present in an earlier published spectrum of HD 187982 (Welter \& Savage 1977).

The $\lambda 6614$ band was investigated at ultra-high resolution using the UHRF instrument on the Anglo-Australian Telescope (AAT) and this led to the discovery of triplet structure in spectra towards $\mu \mathrm{Sgr}$ and $\zeta$ Oph. The shape is reminiscent of the rotational contours of a large molecule - see Fig. 2 (Sarre et al. 1995). The three main components have the appearance of partially resolved R, Q and P branches in the electronic transition of a large molecule. Theoretical work by Cossart-Magos \& Leach (1990) had predicted that electronic transitions of large molecules such as polycyclic aromatic hydrocarbons (PAHs) would produce partially resolved rotational fine structure which is similar to that observed. Similar results for the $\lambda 6614, \lambda 5797$ and $\lambda 6379$ bands were reported in 1996 towards $\zeta$ Oph and two other stars by Ehrenfreund \& Foing (1996).

Rotational contour modelling of the UHRF spectra of the $\lambda 6614$ band was undertaken with results as given in Fig. 3 for a Coriolis-induced ${ }^{1} \mathrm{~B}_{2}-{ }^{1} \mathrm{~A}_{1}$ electronic transition of an oblate symmetric top which has the simplifying characteristic that the value of one 


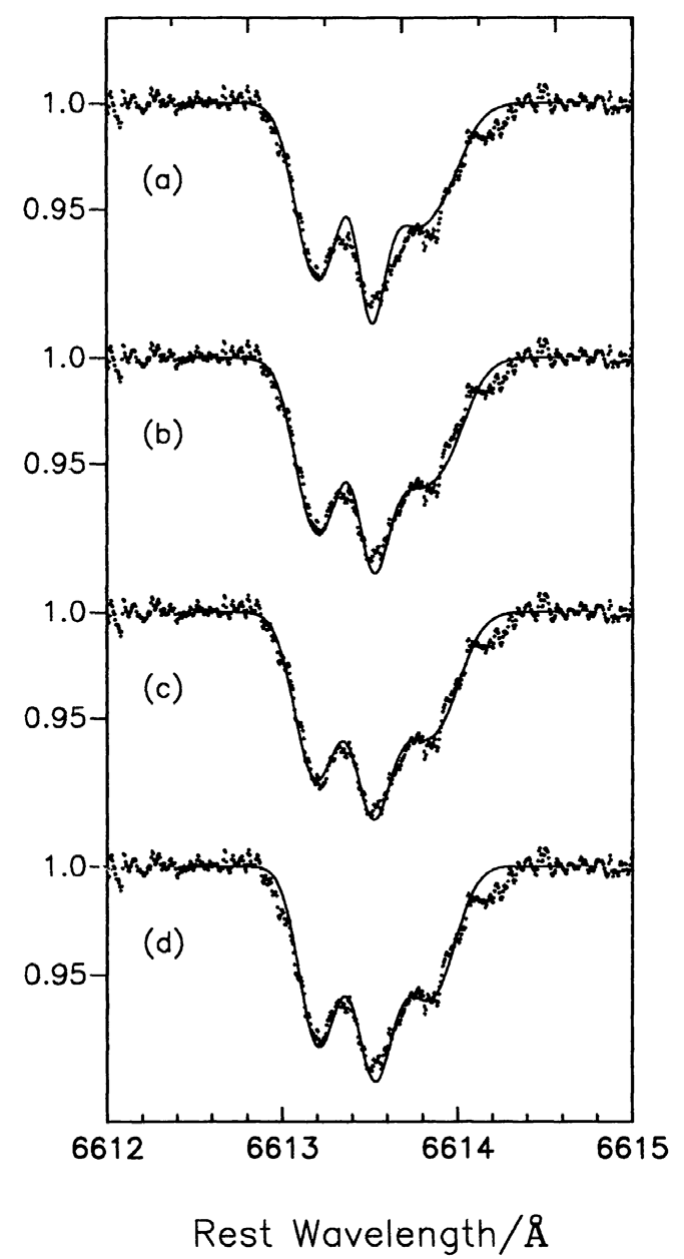

Figure 3. UHRF data for $\lambda 6614$ towards $\mu$ Sgr compared with the results of four calculations with differing starting parameters. Reasonable fits (a)-(d) can be obtained for pairs of $\mathrm{B}^{\prime \prime}$ and $\mathrm{T}_{\text {rot }}$ values which are not readily separated (see text). Reproduced with permission from Kerr et al. 1996.

moment of inertia fixes the other two within the rigid rotor approximation (Kerr et al. 1996). The four examples (a), (b), (c) and (d) correspond to different possible combinations of rotational temperature, $\mathrm{T}_{\text {rot }}$ and rotational constant, $\mathrm{B}$, in the $\chi^{2}$ minimisation calculation. The best fit obtained (c) was for $\mathrm{T}_{r o t}=61 \mathrm{~K}, \mathrm{~B}^{\prime \prime}=0.00336 \mathrm{~cm}^{-1}$ and a $0.17 \%$ reduction in $\mathrm{B}$ on electronic excitation, the other illustrated cases $\left(\mathrm{T} / \mathrm{K}, \mathrm{B}^{\prime \prime} / \mathrm{cm}^{-1}\right.$, $\Delta \mathrm{B} / \%$ ) being (a) 8.9, 0.01913, -0.85, (b) 20.2, 0.00947, -0.42 and (d) 101.3, 0.00286, 0.21 . Given that the $\mathrm{B}$ value for coronene is $c .0 .01 \mathrm{~cm}^{-1}$, the best fit (c) suggests that a somewhat larger $\mathrm{PAH}$ at least the size of circumcoronene $\left(\mathrm{C}_{54} \mathrm{H}_{18}\right)$ with $\mathrm{B} \approx 0.002 \mathrm{~cm}^{-1}$ would be a stronger candidate. Modelling of $\lambda 6614$ has also been reported for molecules of the cumulene carbene type with chemical formula $\mathrm{C}_{n} \mathrm{H}_{2}$ yielding $\mathrm{T}_{\text {rot }}=75 \mathrm{~K}$ for $\mathrm{C}_{12} \mathrm{H}_{2}$ (Schulz et al. 2000).

An interesting issue is the extent to which the exact wavelengths of the components of $\lambda 6614$ may vary with line-of-sight and potentially be related to the rotational temperature of the carrier. This was considered for seven lines-of-sight by Cami et al. (2004) using data of Galazutdinov et al. (2002). It was found that while the wavelength of the 


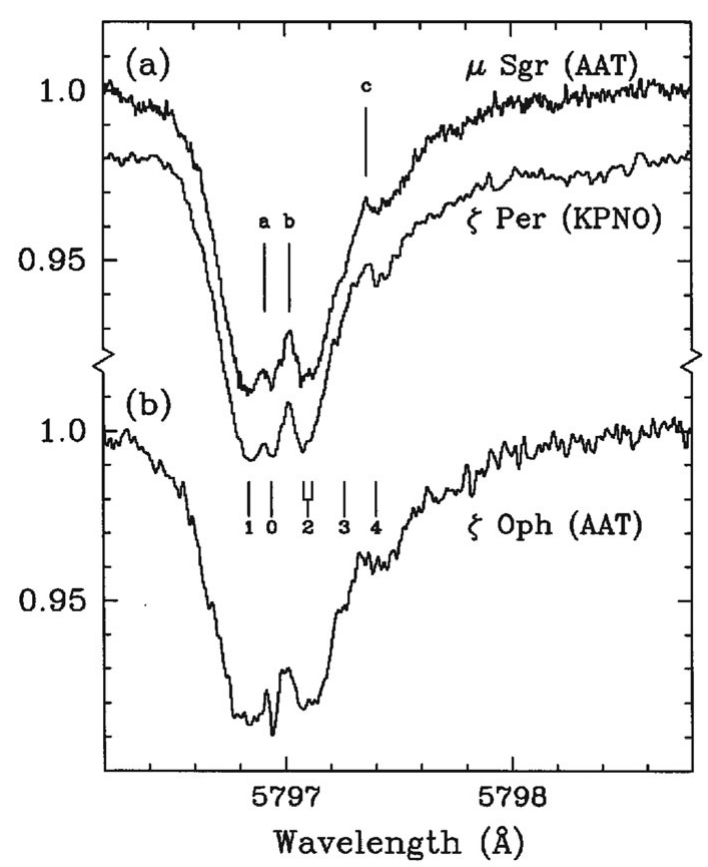

Figure 4. Ultrahigh-resolution spectra of $\lambda 5797$ (a) toward $\mu$ Sgr (AAT) and $\zeta$ Per (KPNO), presented in the rest frames of the main cloud components; and (b) toward $\zeta$ Oph, where the profile has been aligned under the spectra of Fig. 4(a). All spectra are corrected for telluric contamination, rectified, and scaled to the same optical depth at the center of the line. Reproduced with permission from Kerr et al. 1998.

central peak (Peak 2) was invariant, the wavelength separation between outer two peaks (Peaks 1 and 3) varied with line-of-sight, moving to shorter (Peak 1) and longer (Peak 3) wavelength. This observation ruled out an earlier suggestion that the fine structure arises from ${ }^{13} \mathrm{C}$ isotopic variations of a carbon-based molecule (Webster 1996). From the separation between Peaks 1 and 3, it was deduced that the data were consistent with the carrier of $\lambda 6614$ having a $\mathrm{B}$ value of $0.016 \pm 0.003 \mathrm{~cm}^{-1}$ and rotational temperatures between 21 and $26 \mathrm{~K}$ for the various lines-of-sight.

Further studies of many diffuse bands at high resolution have been conducted in recent years (Galazutdinov et al. 2008a,b). These provide an extremely valuable data resource for comparison with the results of modelling and laboratory studies.

\section{Ultra-high-resolution studies of other diffuse bands}

Following the discovery of fine structure in the $6614 \AA$ diffuse band, spectra of $\lambda 5797$ at high signal-to-noise towards $\mu \mathrm{Sgr}, \zeta$ Per and $\zeta$ Oph were obtained using the AAT (UHRF) and KPNO telescopes as shown in Fig. 4 (Kerr et al. 1998). To date no model or laboratory spectra has been able to reproduce the ultrafine structure in this band which remains an exacting test for any proposed carrier(s). An notable feature is the presence of a sharp component labelled ' 0 ' which is reminiscent of a Q-type rotational branch in a molecular electronic transition.

The narrowest of the stronger diffuse bands is $\lambda 6196$. It has been shown to correlate extremely well with $\lambda 6614$ over a large number of sightlines with a correlation coefficient 


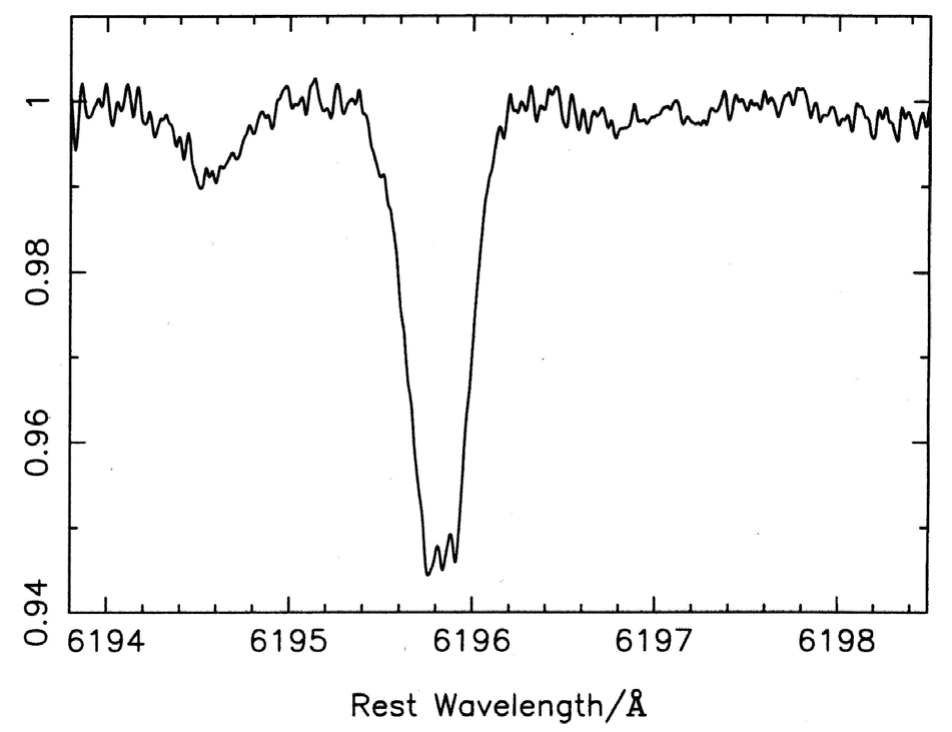

Figure 5. UHRF spectrum of $\lambda 6196$ recorded towards $\mu$ Sgr at RP of 600,000. A triplet of closely spaced lines is present and a blue-degraded tail (see text).

of 0.986 which may suggest a common or very closely related carrier (McCall et al. 2010). UHRF spectra towards $\mu$ Sgr show a narrowly spaced triplet structure which may be due to $\mathrm{P}, \mathrm{Q}$ and $\mathrm{R}$ components as shown in Fig. 5. Confirmation of this structure awaits spectral recordings along other lines-of-sight in a manner comparable to that achieved for $\lambda 5797$ as just described. Overall the $\lambda 6196$ profile is substantially different from that of $\lambda 6614$ and on this basis it is difficult to see how the bands could arise from the same carrier unless they arise from transitions to different electronically excited states.

The UHRF has also been employed to determine whether any fine or ultrafine structure might be resolved in the very broad diffuse band, $\lambda 4430$ (Snow 2002). At a resolving power (RP) of $c .10^{6}$ and including lines-of-sight towards which fine structure had been observed in narrower diffuse bands, no evidence for structure was found in three selected wavelength intervals of the feature, which implies that the broadening of the band is intrinsic. This could arise from rapid internal conversion or rotational line congestion. Related to this aspect is a study published in the same year which investigated the overall profile of $\lambda 4430$ (Snow, Zukowski \& Massey 2002). Although at a modest RP of 2,000, this is quite sufficient for the study. It was found that over 35 lines-of-sight the profile is very well fitted by a Lorentzian function. This is consistent with a lifetime broadening origin and a lifetime of 0.38 ps. The data are given in Fig. 7 of Snow, Zukowski \& Massey (2002) where, it is suggested here, there is evidence for a weak shoulder on the long-wavelength side of the band making it slightly red-degraded - as commonly seen in narrower diffuse band spectra.

\section{Band profile variation}

Building on the initial study of $\lambda 5780$ by Porceddu et al. (1992) in which atypically large widths and redward shifts for this band were found towards Orion Trapezium stars, Galazutdinov et al. (2008b) have reported blue-shifted diffuse bands in the Sco OB1 association. This may reflect differing internal temperatures for the carriers as is also found in the study by Kaźmierczak et al. (2010) where the widths and shapes of $\lambda 6196$ 
and $\lambda 5797$ appear to depend on the kinetic and internal rotational temperature of the $\mathrm{C}_{2}$ molecule observed along the same lines-of-sight. In the most significant advance in the field for some years, dramatically different profiles have been discovered for some diffuse bands towards Herschel 36 as described elsewhere in this volume (Dahlstrom et al. and Oka et al.). The origin of the change appears to be heating of the carriers by a nearby infrared source.

\section{Summary}

The profiles of many of the diffuse interstellar bands are now quite well defined. This constitutes an important step in stimulating new ideas among researchers and new tests for proposed carriers based on laboratory and theoretical work. The primary characteristics of central wavelength and width remain important but are now supplemented by observations of high calibre that provide much more rigorous criteria for assessing any claim of assignment. A notable feature, alongside the sheer number of diffuse bands, is the considerable variation in diffuse band profiles; both of these aspects are readily accommodated within the molecular hypothesis in which the solution will surely be found.

\section{Acknowledgements}

We thank PATT for the award of telescope time and The University of Nottingham and EPSRC for financial support. The data in figure 5. were obtained in an observing run with Robert Hibbins.

\section{References}

Bromage, G. E. 1987, QJRAS, 28, 294

Cami, J., Salama, F., Jiménez-Vicente, J., et al. 2004, ApJL, 611, L113

Cossart-Magos, C. \& Leach, S. 1990, A\& A, 233, 559

Danks, A. C. \& Lambert, D. L. 1976, MNRAS, 174, 571

Ehrenfreund, P. \& Foing, B. H. 1996, A\& A, 307, L25

Galazutdinov, G., Moutou, C., Musaev, F., \& Krełowski, J. 2002, AAP, 384, 215

Galazutdinov, G. A., LoCurto, G., \& Krełowski, J. 2008, ApJ, 682, 1076

Galazutdinov, G. A., LoCurto, G., \& Krełowski, J. 2008a, MNRAS, 386, 2003

Galazutdinov, G. A., LoCurto, G., Han, I., \& Krełowski, J. 2008b, PASP, 120, 178

Herbig, G. H. 1975, ApJ, 196, 129

Herbig, G. H. \& Soderblom, D. R. 1982, ApJ, 252, 610

Kaźmierczak, M., Schmidt, M. R., Galazutdinov, G. A., et al. 2010, MNRAS, 408, 1590

Kerr, T. H., Hibbins, R. E., Miles, J. R., et al. 1996, MNRAS, 283, L105

Kerr, T. H., Hibbins, R. E., Fossey, S. J., Miles, J. R., \& Sarre, P. J. 1998, ApJ, 495, 941

McCall, B. J. \& Griffin, R. E. 2013, Proc. Roy. Soc. A, 469, 201206

McCall, B. J., Drosback, M. M., Thorburn, J. A., et al. 2010, ApJ, 708, 1628

Merrill, P. W. 1934, PASP, 46, 206

Porceddu, I., Benvenuti, P., \& Krełowski, J. 1992, PASP, 260, 391

Sarre, P. J., Miles, J. R., Kerr, T. H., et al. 1995, MNRAS, 277, L41

Savage, B. D. 1976, ApJ, 205, 122

Schulz, S. A., King, J. E., \& Glinski, R. J. 2000, MNRAS, 312, 769

Snow, T. P., Zukowski, D., \& Massey, P. 2002, ApJ, 587, 877

Snow, T. P. 2002, ApJ, 567, 407

Webster, A. 1996, MNRAS, 282, 1372

Welter, G. L. \& Savage, B. D. 1977, ApJ, 215, 788 